\title{
Toward New Classification Criteria for Juvenile Idiopathic Arthritis: First Steps, Pediatric Rheumatology International Trials Organization International Consensus
}

\author{
Alberto Martini, Angelo Ravelli, Tadej Avcin, Michael W. Beresford, Ruben Burgos-Vargas, \\ Ruben Cuttica, Norman T. Ilowite, Raju Khubchandani, Ronald M. Laxer, Daniel J. Lovell, \\ Ross E. Petty, Carol A. Wallace, Nico M. Wulffraat, Angela Pistorio, and Nicolino Ruperto, \\ for the Pediatric Rheumatology International Trials Organization (PRINTO)
}

\begin{abstract}
Objective. To revise the current juvenile idiopathic arthritis (JIA) International League of Associations for Rheumatology (ILAR) classification criteria with an evidence-based approach, using clinical and routine laboratory measures available worldwide, to identify homogeneous clinical groups and to distinguish those forms of chronic arthritis typically seen only in children from the childhood counterpart of adult diseases.

Methods. The overall project consists of 4 steps. This work represents Step 1, a Delphi Web-based consensus and Step 2, an international nominal group technique (NGT) consensus conference for the new provisional Pediatric Rheumatology International Trials Organization JIA classification criteria. A future large data collection of at least 1000 new-onset JIA patients (Step 3) followed by analysis and NGT consensus (Step 4) will provide data for the evidence-based validation of the JIA classification criteria.

Results. In Step 1, three Delphi rounds of interactions were implemented to revise the 7 ILAR JIA categories. In Step 2, forty-seven questions with electronic voting were implemented to derive the new proposed criteria. Four disorders were proposed: (a) systemic JIA; (b) rheumatoid factor-positive JIA; (c) enthesitis/spondylitis-related JIA; and (d) early-onset antinuclear antibody-positive JIA. The other forms were gathered under the term "others." These will be analyzed during the prospective data collection using a list of descriptors to see whether the clustering of some of them could identify homogeneous entities.

Conclusion. An international consensus was reached to identify different proposed homogeneous chronic disorders that fall under the historical term JIA. These preliminary criteria will be formally validated with a dedicated project. (First Release October 1 2018; J Rheumatol 2019;46:190-7; doi:10.3899/jrheum.180168)
\end{abstract}

Key Indexing Terms:

JUVENILE IDIOPATHIC ARTHRITIS

AUTOIMMUNE DISEASES

ANTINUCLEAR ANTIBODIES

\begin{abstract}
From Istituto di Ricovero e Cura a Carattere Scientifico (IRCCS) Istituto Giannina Gaslini, Direzione Scientifica; IRCCS Istituto Giannina Gaslini, Clinica Pediatrica e Reumatologia, Pediatric Rheumatology International Trials Organization (PRINTO), and Università degli Studi di Genova; IRCCS Istituto Giannina Gaslini, Servizio di Epidemiologia e Biostatistica, Genoa, Italy; University Children's Hospital, University Medical Center Ljubljana, Department of Allergology, Rheumatology and Clinical Immunology, Ljubljana, Slovenia; Department of Paediatric Rheumatology, Alder Hey Children's National Health Service (NHS) Foundation Trust; Institute of Translational Medicine, University of Liverpool, Liverpool, UK; Hospital General de Mexico, Departamento de Reumatología, Mexico City, Mexico; Hospital Pedro de Elizalde, Rheumatology Section, Buenos Aires, Argentina; Children's Hospital at Montefiore, Albert Einstein College of Medicine, Pediatrics, New Hyde Park, New York; Cincinnati Children's Hospital Medical Center, Division of Rheumatology, Cincinnati, Ohio; Seattle Children's Hospital, Seattle, Washington, USA; Jaslok Hospital and Research Centre, Department of Paediatrics, Mumbai, India; The Hospital for Sick Children, Division of
\end{abstract}

\begin{abstract}
Rheumatology, Department of Paediatrics, University of Toronto, Toronto, Ontario; British Columbia Children's Hospital, Department of Pediatrics, University of British Columbia, Vancouver, British Columbia, Canada; Wilhelmina Children's Hospital, Department of Pediatric Immunology and Rheumatology, Utrecht, the Netherlands.

This project has been funded by the PRINTO research funding at the IRCCS Istituto Giannina Gaslini, Genoa, Italy. No external entity such as pharmaceutical companies has been involved at any stage of the project.

A. Martini, MD, Professor, IRCCS Istituto Giannina Gaslini, Direzione Scientifica; A. Ravelli, MD, Professor, IRCCS Istituto Giannina Gaslini, Clinica Pediatrica e Reumatologia and Università degli Studi di Genova; T. Avcin, MD, PhD, University Children's Hospital, University Medical Center Ljubljana, Department of Allergology, Rheumatology and Clinical Immunology; M.W. Beresford, MBChB, PhD, Department of Paediatric Rheumatology, Alder Hey Children's NHS Foundation Trust, and Institute of Translational Medicine, University of Liverpool; R. Burgos-Vargas, MD, Hospital General de Mexico, Departamento de Reumatología;
\end{abstract}


Juvenile idiopathic arthritis (JIA) is a diagnosis of exclusion that encompasses all forms of chronic arthritis of unknown origin, starting before 16 years of age. The most recent classification proposed in 1995 by a consensus based on expert opinions of pediatric rheumatologists representing each of the leagues of the International League of Associations for Rheumatology (ILAR) consists of 6 different, mutually exclusive categories defined in clinical and laboratory measures: systemic arthritis, oligoarthritis (persistent or extended), polyarthritis rheumatoid factor (RF)-positive, polyarthritis RF-negative, enthesitis-related arthritis (ERA), psoriatic arthritis (PsA), and a seventh category, undifferentiated arthritis, which includes those patients who do not fit any criteria or fit more than one ${ }^{1,2}$.

Since then, increasing evidence has accumulated suggesting that while some of these categories appear to be quite homogeneous and present both in children as well as in adults, others are heterogeneous and may be better defined ${ }^{3,4}$. Systemic JIA is characterized by an autoinflammatory phenotype (fever, rash, lymphadenopathies, marked systemic inflammation, etc.), with its adult equivalent named adult-onset Still disease (AOSD). RF-positive polyarthritis represents the childhood equivalent of RF-positive adult rheumatoid arthritis (RA), and enthesitis-related arthritis is a form of undifferentiated spondyloarthritis (SpA). On the other hand, 2 categories, RF-negative polyarthritis and PsA, have been shown to be heterogeneous. They both include a subset of patients characterized by early onset and antinuclear antibody (ANA) positivity, a more homogeneous entity observed only in childhood, representing the majority of patients with oligoarticular JIA in the Western world ${ }^{3,5,6}$. It seems, therefore, that the number of joints involved and the presence of psoriasis may not represent reliable criteria for JIA classification.

There is growing impetus to harmonize as much as possible the criteria in use for those diseases that are observed both in children and in adults either for use in clinical trials, in research, or in the everyday clinical setting $4,7,8,9,10,11$. The

R. Cuttica, MD, Hospital Pedro de Elizalde, Rheumatology Section; N.T. Ilowite, MD, Children's Hospital at Montefiore, Albert Einstein College of Medicine, Pediatrics; R. Khubchandani, MD, Jaslok Hospital and Research Centre, Department of Paediatrics; R.M. Laxer, MD, The Hospital for Sick Children, Division of Rheumatology, Department of Paediatrics, University of Toronto; D.J. Lovell, MD, MPH, Cincinnati Children's Hospital Medical Center, Division of Rheumatology; R.E. Petty, $M D, P h D$, British Columbia Children's Hospital, Department of Pediatrics, University of British Columbia; C.A. Wallace, MD, Seattle Children's Hospital; N.M. Wulffraat, MD, PhD, Wilhelmina Children's Hospital, Department of Pediatric Immunology and Rheumatology; A. Pistorio, MD, PhD, IRCCS Istituto Giannina Gaslini, Servizio di Epidemiologia e Biostatistica; N. Ruperto, MD, MPH, IRCCS Istituto Giannina Gaslini, Clinica Pediatrica e Reumatologia, PRINTO.

Address correspondence to Prof. A. Martini, Scientific Director, IRCCS Istituto Giannina Gaslini, Via G. Gaslini 5, 16147 Genoa, Italy.

E-mail:albertomartini@gaslini.org

Full Release Article. For details see Reprints and Permissions at jrheum.org Accepted for publication June 22, 2018. aims of this project, conducted by the Pediatric Rheumatology International Trials Organization (PRINTO; www.printo.it) were the following: (1) to propose revision of the current JIA ILAR classification criteria with an evidence-based approach, using clinical and routine laboratory measures available worldwide; and (2) to distinguish those forms of chronic arthritis that are typically seen in children from those that represent the childhood counterpart of diseases observed in adults.

\section{MATERIALS AND METHODS}

A multistep process strategy has been set up to derive the new JIA PRINTO classification criteria, which included the following steps: (1) a Web-based Delphi consensus survey among 13 international pediatric rheumatologists plus 1 consensus moderator (NR) and a biostatistician (AP), aiming to propose revision of the current ILAR JIA classification criteria; (2) a nominal group technique (NGT) consensus conference to discuss and propose the new JIA PRINTO classification criteria; (3) a large-scale data collection of patients with new-onset JIA for the validation of the proposed criteria; and (4) a final NGT consensus conference to discuss the results of the validation step (Figure 1).

Consensus formation techniques. Two well-recognized consensus formation methodologies, specifically designed to combine judgments from a group of experts in a particular field, were used to attain the goals: the Delphi Technique and NGT ${ }^{12,13}$. The Delphi Technique uses a series of well-defined questionnaires, while NGT is a structured face-to-face meeting designed to facilitate reaching consensus, through round-robin discussion (the first to speak was randomly selected followed by all remaining panelists) ${ }^{14-22}$. A consensus of 10/13 (76.9\%) of the panelists was required for each step. A dedicated Web application was developed by the PRINTO staff (RC, LV, $\mathrm{CP})$ to manage consensus with electronic voting ${ }^{13}$.

Step 1: Delphi Web-based consensus. The international panel was asked to review the relevant literature and to propose changes to the current ILAR criteria. The Delphi process involved several rounds of Web-based interaction wherein the first round opened with participants working independently from the others. For all the following rounds, a Web-based discussion was implemented to share the comments among all the participants.

Step 2: NGT consensus conference. An NGT consensus conference was held in Genoa, Italy, on December 4-5, 2015, to discuss and debate in person in a structured round robin and finalize the proposed new JIA classification criteria.

The first 2 steps are the subject of this manuscript while the other 2 are currently in the implementation phase (Supplementary Data 1 and 2, available with the online version of this article).

\section{RESULTS}

Step 1: Delphi Web-based consensus. Three Delphi Web rounds of interactions were implemented. Participants were asked to comment and discuss in a written fashion a general definition for JIA and a definition for each of the 7 ILAR JIA categories. Participants were asked to revise the Word documents in Track Changes and provide revisions/criticisms/ comments on the current ILAR JIA classification criteria, while taking into account the comments from the other participants. The panel consensus was below the required threshold of $80 \%$ as follows: general definition of JIA (69\%), systemic JIA (51\%), oligoarthritis (42\%), polyarthritis RF-negative (38\%), polyarthritis RF-positive (58\%), PsA (69\%), ERA (39\%), and undifferentiated arthritis (69\%).

Personal non-commercial use only. The Journal of Rheumatology Copyright $\odot$ 2019. All rights reserved. 


\begin{tabular}{|c|}
\hline Delphi web-based consensus \\
in 2015 \\
\hline
\end{tabular}

STEP 2: Nominal Group Technique Consensus (this manuscript)

\begin{tabular}{|c|}
\hline First consensus conference \\
held in Genoa, Italy on \\
December 4-5, 2015 \\
\hline
\end{tabular}

STEP 3: Data collection and validation (future work)

\begin{tabular}{|c|}
\hline Prospective data collection of \\
at least 1000 new onset \\
children with JIA followed \\
prospectively for 1-5 years
\end{tabular}

STEP 4: Nominal Group Technique Consensus (future work)

\begin{tabular}{|c|}
\hline $\begin{array}{c}\text { Second and final consensus } \\
\text { conference }\end{array}$ \\
\hline
\end{tabular}

Figure 1. Flow chart of the overall project. The first 2 steps are the subject of this manuscript, while the last 2 are related to the validation procedures of the proposed criteria. Children mentioned in Step 3 will be those with biosamples. JIA: juvenile idiopathic arthritis.

Step 2: NGT consensus conference. There were 139 planned questions for discussion; of these, $47 / 139$ (33.8\%) were actually discussed and voted on, while all remaining questions were discarded (Table 1). Most of the 47 discussion rounds were devoted to the general definitions of JIA $(29.8 \%)$, systemic JIA (25.5\%), and enthesitis/spondylitis-related JIA (19.1\%).

Two additional Web rounds were done postconsensus conference for a further refinement of the enthesitis/spondylitis-related JIA and of early-onset ANA-positive JIA, with $100 \%$ consensus achieved.

General definition of JIA. JIA comprises a group of inflammatory disorders that begins before the 18th birthday and persists for at least 6 weeks (must fulfill 1 of the A-E criteria below); other known conditions are excluded. 
Table 1. Details on nominal group technique voting sessions during the 2-day consensus conference.

\begin{tabular}{lccc}
\hline Voting Sessions & $\begin{array}{c}\text { No. Planned } \\
\text { Voting Sessions* } \\
\mathrm{n}=139\end{array}$ & $\begin{array}{c}\text { No. Final } \\
\text { Voting Sessions, } \\
\mathrm{n}=47\end{array}$ & $\begin{array}{c}\text { No. Voting } \\
\text { Sessions with } \\
\text { Consensus, } \mathrm{n}=47\end{array}$ \\
\hline Final voting sessions & $47(33.8)$ & & \\
General definition of JIA & & $14(29.8)$ & $13(93)$ \\
$\quad$ Systemic JIA & $12(25.5)$ & $5(41.7)$ \\
RF-positive JIA & $3(6.4)$ & $2(66.7)$ \\
Enthesitis/spondylitis-related JIA & $9(19.1)$ & $5(55.6)$ \\
Early-onset ANA-positive JIA & $2(4.3)$ & $2(100)$ \\
Other JIA & $1(2.1)$ & $1(100)$ \\
$\quad$ Unclassified JIA & $1(2.1)$ & $1(100)$ \\
Other rounds of discussion & & $1(2.1)$ & $1(100)$ \\
Age at onset $<18$ yrs & & $2(4.3)$ & $1(50)$ \\
Juvenile PsA & $2(4.3)$ & $1(50)$ \\
Asymmetric arthritis & & \\
Other planned voting sessions discarded & & & Discarded \\
and not discussed* & $92(66.1)$ & & \\
\hline
\end{tabular}

Data are $\mathrm{n}(\%)$ * The planned electronic voting sessions were prepared by the organizers in excess of the real needs to cover all potential discussion topics. JIA: juvenile idiopathic arthritis; RF: rheumatoid factor; ANA: antinuclear antibody; PsA: psoriatic arthritis.

Comment: Although the term JIA could generate the misleading concept that it represents a single disease, it was decided to maintain the general term to keep with the literature and terminology accepted by the regulatory authorities (US Food and Drug Administration, and European Medicines Agency). JIA is preceded by an adjective that characterizes the reference to the distinct disorder (e.g., systemic JIA). The term group was used to better underline the concept that JIA is not a single disease but rather a group of different disorders. The term disorder (instead of ILAR categories) has been implemented to further underline the concept that JIA is not a single disease. The presence of arthritis is no longer included because, similar to the criteria for AOSD, patients without arthritis have been included in the definition of systemic JIA. Therefore, the presence of arthritis of $\geq 6$ weeks of duration is now mentioned in the pertinent criteria. In addition, the onset of the disease has been changed to before 18 years of age to distinguish children/adults, as adopted by many countries.

Supplementary Data 1 (available with the online version of this article) reports the definition of each term and the list of additional JIA descriptors to be collected during the validation process and analysis, as described in Supplementary Data 2.

A. Systemic JIA: definition. Fever of unknown origin (excluding infectious, neoplastic, autoimmune, or monogenic autoinflammatory diseases) that is documented to be daily (quotidian; fever that rises to $\geq 39^{\circ} \mathrm{C}$ once a day and returns to $\leq 37{ }^{\circ} \mathrm{C}$ between fever peaks) for at least 3 consecutive days and reoccurring over a duration of at least 2 weeks and accompanied by 2 major criteria OR 1 major criterion and 2 minor criteria. Major criteria are (1) evanescent (nonfixed) erythematous rash; and (2) arthritis. Minor criteria are (1) generalized lymph node enlargement and/or hepatomegaly and/or splenomegaly; (2) serositis; (3) arthralgia lasting 2 weeks or longer (in the absence of arthritis); and (4) leukocytosis $\left(\geq 15,000 / \mathrm{mm}^{3}\right)$ with neutrophilia.

Comment: Systemic arthritis (systemic JIA) was considered to be equivalent to AOSD; therefore, a modified version of the Yamaguchi criteria has been chosen. The duration of cutoff for arthralgia is that of the Yamaguchi criteria, but the cutoff for leukocytosis is greater $\left(15,000 / \mathrm{mm}^{3}\right.$ vs $10,000 / \mathrm{mm}^{3}$ ), reflecting the clinical knowledge of this disease among the panelists ${ }^{23}$. The proposed criteria rely primarily on the presence of fever (daily spikes) in line with the ILAR definition. The term fever reoccurring has been introduced to underline that within the 2-week timeframe, fever could be present for some days (at least 3) and absent in other days. In addition to fever, the presence of at least 1 major criterion is necessary, while arthritis is no longer required because, as in adults, it is well known that there are patients who might lack arthritis but have the same systemic features seen in classic systemic JIA. Similarly, the duration of arthritis is not specified because it is very likely that in current practice, a child with systemic JIA and arthritis will be treated before 6 weeks of arthritis have elapsed. No consensus was reached by the proposal to change the name of this condition into Still disease, because the current trend is to avoid the use of eponyms. There was consensus to keep the term systemic JIA, even though some patients may not have arthritis, to be consistent with the current accepted terminology. Similarly, there was consensus to keep systemic JIA among the JIA disorders rather than grouping it with autoinflammatory diseases.

B. RF-positive JIA.

- Arthritis for $\geq 6$ weeks, and 
- Association with 2 positive tests for RF at least 3 months apart or at least 1 positive test for antibodies to cyclic citrullinated peptide (CCP).

Comment: RF-positive JIA was considered to be the equivalent of adult RF-positive RA. Because the number of joints involved is no longer a classification criterion, arthritis was used instead of polyarthritis. The duration of arthritis for $\geq 6$ weeks is reported in the criteria to differentiate the chronicity as opposed to arthritis of other origin such as reactive arthritis, which is usually self-limiting. Along with the presence of arthritis, the association with at least 2 positive tests for RF (to avoid spurious findings) has been maintained; the proper cutoffs for RF will come from the validation phase, when a central laboratory will be used to derive positivity. In addition, the positivity to antibodies to CCP is now considered a valid alternative.

\section{Enthesitis/spondylitis-related JIA.}

- Peripheral arthritis and enthesitis, or

- Arthritis or enthesitis, plus $\geq 3$ months of inflammatory back pain and sacroiliitis on imaging, or

- Arthritis or enthesitis plus 2 of the following: (1) sacroiliac joint tenderness; (2) inflammatory back pain; (3) presence of HLA-B27 antigen; (4) acute (symptomatic) anterior uveitis; and (5) history of a $\mathrm{SpA}$ in a first-degree relative.

If peripheral arthritis is present, it should persist for at least 6 weeks.

Comment: Enthesitis-related JIA was considered an undifferentiated SpA. All the different forms of adult SpA can be found in children, with the major difference being the higher proportion of undifferentiated forms in children. The current definition has been partly harmonized with the adult one, and an imaging criterion for radiographs ${ }^{24}$ or magnetic resonance imaging $^{25}$ has been introduced (Supplementary Data 1, available with the online version of this article). The definition of Sieper, et $a l^{26}$ of inflammatory back pain has been adopted. Because the term ERA could wrongly suggest the existence of a form of SpA that is specific to childhood, it was initially proposed to name this condition juvenile $\mathrm{SpA}$ and was later changed to enthesitis/spondylitis-related JIA. This disorder required a postconsensus Web discussion, which was agreed to by all 13 panelists.

D. Early-onset ANA-positive JIA.

- Arthritis for $\geq 6$ weeks, and

- Early-onset ( $\leq 6 \mathrm{yrs})$, and

- Presence of 2 positive ANA tests with a titer $\geq 1 / 160$ (tested by immunofluorescence) at least 3 months apart.

Exclusions are systemic JIA, RF-positive arthritis, and enthesitis/spondylitis-related JIA.

Comment: This condition represents a new entry and its definition is based on previous work that has supported the identification of a homogeneous form of arthritis characterized by early onset, female predominance, high incidence of chronic iridocyclitis, ANA positivity, and consistent HLA association ${ }^{3,5,6}$. This form appears to exist only in children and does not have a counterpart in adult patients. In Western countries, this is the most frequent form of JIA and represents the vast majority of patients previously classified in the oligoarticular category. In addition it has been shown that this same entity was previously included in the RF-negative polyarthritis category (if $>4$ joints were involved in the first 6 months of the disease) or in the PsA category (if psoriasis or same psoriatic features were present) $)^{3,5,6}$. The age cutoff is also supported by the work of Barnes, et al, who studied gene expression in oligoarticular and polyarticular JIA and found that patients with early-onset arthritis ( $\leq 6 \mathrm{yrs}$ ) are characterized by a B cell signature independent of the number of joints involved ${ }^{27}$.

Because ANA can occasionally be positive in healthy populations and in other conditions, the following have been introduced just for this specific entity to exclude the spurious positivity of ANA in the other JIA disorders: (a) a titer cutoff of $\geq 1 / 160$ on immunofluorescence on HEp-2 cells on 2 occasions at least 3 months apart (this cutoff has proved to perform well in previous studies $\left.{ }^{5,6}\right)$; and (b) exclusion criteria.

This disorder required a postconsensus Web discussion, which was agreed to by all 13 panelists.

\section{E. Other JIA.}

- Arthritis for $\geq 6$ weeks

- Does not fit criteria for disorders A to D.

Comment: While there was consensus that disorders from A to $\mathrm{D}$ represent defined entities, no consensus could be reached on criteria that identify other well-characterized diseases. It was therefore decided to provisionally lump together all the other forms of JIA and to postpone the potential identification of other entities until after the analysis of the data of the prospective validation, when patients belonging to the "other JIA" category will be analyzed using a long list of descriptors to see whether the clustering of some of them could identify homogeneous entities. In particular, no consensus was reached on juvenile PsA, whose definition was postponed to the end of the prospective data collection to identify the correct descriptor of this JIA disorder. "Other JIA" therefore represents a provisional solution waiting for better classification evidence provided by the analysis of the prospective data collection.

F. Unclassified JIA.

- Arthritis for $\geq 6$ weeks

- Fits $>1$ disorder A-D.

Comment: This is a provisional residual category in case, during the prospective data collection, some patients fit $>1$ of the disorders described by criteria above (A-D disorders). Data will be analyzed in phase 2 of the project at the end of the data collection.

Outlook and outstanding future work. Figure 1 reports the

Personal non-commercial use only. The Journal of Rheumatology Copyright @ $\odot$ 2019. All rights reserved 
following steps necessary for the evidence-based final validation of the consensus criteria.

In Step 3, it is foreseen that a prospective cohort of 1000 patients with JIA at onset will be collected, with a longitudinal followup for $1-5$ years.

In Step 4, once the prospective data collection is finished and analyzed, a final consensus conference with NGT will be organized to discuss the results and to finalize the new, validated PRINTO JIA classification criteria. The full methodological details for Steps 3 and 4 are reported in Supplementary Data 2 (available with the online version of this article).

\section{DISCUSSION}

The current ILAR classification criteria for JIA ${ }^{1,2}$, developed by consensus and not formally validated, represent an important advance because they provide a single classification scheme accepted worldwide. However, criticisms have been raised, including that some ILAR categories do not appear to identify homogeneous entities. Those criticisms were not addressed in the revisions of the ILAR criteria ${ }^{3,4}$. Here, we report the first steps of a process aimed at providing new evidence-based classification of JIA and present a formal process by which these preliminary criteria will be validated in the next few years.

This first step reflects the effort to identify, based on worldwide available clinical and laboratory measures, distinct and homogeneous disorders characterized by chronic childhood arthritis, and to set the stage for the validation of the proposed criteria. The disorders provisionally classified as "others" during the first step will be better characterized during the prospective data collection from at least 1000 new-onset patients.

The identified disorders are considered the juvenile counterparts of diseases also observed in adults (systemic JIA, RF-positive JIA, enthesitis/spondylitis-related JIA) or are typical only of children (early-onset ANA-positive JIA).

Systemic JIA was considered equivalent to AOSD, representing about 10-15\% of all JIA cases, while AOSD is rarer. In fact, these 2 conditions share a lot of strong similarities such as the clinical and biological features, including the occurrence of macrophage activation syndrome, and the striking response to interleukin 1 (IL-1) and IL-6 inhibitors ${ }^{28,29}$. Given the marked activation of the innate immune system as well as the important pathogenic role played by IL- 1 and IL-6, this condition is considered to be a polygenic autoinflammatory disease ${ }^{30}$. Probably, as is the case for monogenic autoinflammatory diseases, patients with systemic JIA represent not a disease, but rather a syndrome, characterized by a marked and persistent activation of the innate immune system. This potential heterogeneity is suggested by the exquisite sensitivity to IL-1 blockade presented by a subset of patients as well as by differences in clinical course ${ }^{31,32}$. Indeed, in about half of the patients, the disease is characterized by relapses followed by intervals of remission with little arthritis, while the other half of the disease follows an unremitting course with persistent arthritis. Moreover, there are patients who present exactly the same clinical and biological systemic features observed in systemic JIA but who never develop arthritis, and therefore cannot by definition be classified as having systemic JIA under the current ILAR classification. This subgroup of patients presently lacks any taxonomic definition in children, although they are included in the definition of AOSD using Yamaguchi criteria, with minor modifications ${ }^{23}$.

RF-positive arthritis was considered the pediatric counterpart of adult RF-positive RA. Several aspects support this assumption: (1) the clinical phenotype is the same and is characterized by female predominance, late onset, and a symmetric arthritis affecting principally wrists and the small joints of the hands and feet; (2) it usually has a progressive course and indeed, among the various JIA categories, it is the one with the worst prognosis ${ }^{33}$; (3) it is the only JIA category in which positive antibodies to CCP have been found ${ }^{34,35}$; and (4) it shares with adult RF-positive RA the same HLA associations $^{7}$. At variance with adults, it represents just a small proportion (around 5\%) of childhood chronic arthritis. Given their high specificity, anti-CCP antibodies were included in the new proposed criteria ${ }^{36}$.

ERA is considered the pediatric counterpart of adult ankylosing spondylitis. It is characterized by the following: (1) the presence of enthesitis; (2) the presence of axial skeletal involvement (although sacroiliac joint involvement is of rarer occurrence than in adults, it is observed in a variable proportion of patients, usually later in disease course); (3) a strong association with HLA-B27; (4) the occurrence of acute anterior uveitis; and (5) a frequent positive familial history for $\mathrm{SpA}^{37,38,39}$. It accounts for about $5-10 \%$ of all JIA cases, with a higher prevalence in non-Western European countries. Therefore, although in children the disease usually presents as an undifferentiated form of $\mathrm{SpA}$, it does not show features different from those observed in adults. To better clarify this aspect, the disease was renamed enthesitis/spondylitis-related JIA.

A new disorder has been suggested by the proposed criteria and called early-onset ANA-positive JIA. This appears to represent a condition unique to the pediatric population and includes up to $50 \%$ of all JIA cases. In the previous ILAR oligoarthritis category, the large majority of patients was characterized by several common features, suggesting a well-defined disease entity: an asymmetric arthritis, an early onset ( $<6$ yrs of age), a female predominance, positive ANA, a high risk for developing chronic iridocyclitis, and consistent HLA associations. It was observed that patients with these same common features were also classified as having RF-negative polyarthritis because of differences in the spread of arthritis and in the number of affected joints, or as PsA because of the presence of psoriasis

Personal non-commercial use only. The Journal of Rheumatology Copyright @ 2019 . All rights reserved. 
or of some psoriatic features ${ }^{3,5,6}$. It was therefore considered advisable to group all these in the new category of early-onset, ANA-positive arthritis, independent of the number of joints involved or the presence of psoriasis.

There was no consensus to identify other homogeneous entities. It has been recognized that the number of joints involved, as well as the presence of psoriasis, may not be useful in children to define homogeneous entities $3,4,5,6$. Therefore, it was decided to group as "other JIA disorders" all those forms of arthritis that do not fit the criteria of the 4 diseases mentioned above. A number of descriptors have been defined to try to identify, on objective criteria, other potential homogeneous entities during the prospective data collection. These entities will presumably include patients with a late-onset ANA-negative symmetric polyarthritis, a condition similar to the one observed in adults or with other forms of spondyloarthropathy such as PsA. Therefore, "other JIA" represents a provisional entity instrumental to characterizing different disorders with an evidence-based approach based on the clustering of a set of descriptors.

As mentioned, except those for the definition of early-onset ANA-positive arthritis, there are no exclusion criteria in the current proposed classification. Therefore, in these provisional PRINTO criteria, the 5 ILAR exclusion criteria are not being used because they were the principal reason why many patients were classified in the ILAR classification as having "undifferentiated arthritis."

The literature now provides some initial evidence that genetic analysis $7,8,9,10,11$ might help to provide additional clues for classification of homogeneous entities. In the validation phase we have planned to collect and store DNA for future genetic analysis. However, there was agreement within the panel that at the current stage, clinical and laboratory criteria available worldwide should be the bases of the new validated criteria, to allow their use in the research setting and in everyday clinical practice worldwide.

We could not proceed with validation of the proposed new criteria in existing cohorts [including the PRINTO, Research in Arthritis in Canadian Children Emphasizing Outcomes (ReACCh-Out) cohort, the Nordic cohort, the German Inception Cohort of Newly diagnosed patients with JIA (ICON-JIA), or the British Childhood Arthritis Prospective Study (CAPS) cohort] because at present they are all lacking 1 or more components of the proposed new criteria such as ANA testing. For taxonomy, it was initially proposed to change the nomenclature to harmonize it with the adult diseases, but consensus was not reached. The issue of final nomenclature will be discussed again in an evidence-based fashion at the end of the validation phase after the collection of the planned 1000 patients. Official endorsement for the future criteria will be sought from learned societies, such as the Pediatric Rheumatology European Society, the American College of Rheumatology, and the European League Against Rheumatism.
A process has been initiated to provide evidence-based, validated new criteria to identify the various diseases included under the umbrella term JIA. The aim is to identify homogeneous entities and to distinguish those disorders that are observed both in children and in adults from those observed only in children. This distinction is of fundamental importance because for similar diseases in children and adults, efficacy trials in children should not be necessary, and pharmacokinetic/pharmacodynamic and safety data could be enough for registration. After literature review, an international consensus was reached to identify 4 different chronic disorders that fall under the historical term JIA; 3 of them are the childhood counterpart of diseases while 1 represents a form of arthritis that is unique and limited to children. The criteria of these 4 entities will be validated during the prospective data collection. Rather than developing new hypothetical criteria instead of the number of joints involved or the presence of psoriasis, it was decided to group all the other forms of arthritis in the group of "other JIA." Data collected prospectively on new-onset patients falling under the definition of "other JIA" will be analyzed according to a set of descriptors (age at onset, asymmetric or symmetric involvement, etc.) in the hypothesis that some of these descriptors will cluster together and define, with an evidence-based approach, new homogeneous entities.

\section{ACKNOWLEDGMENT}

We are grateful to the PRINTO staff for the implementation and management of the consensus phases processed through an electronic voting system developed by Roberto Cavanna, Luca Villa, and Chiara Pallotti.

\section{ONLINE SUPPLEMENT}

Supplementary material accompanies the online version of this article.

\section{REFERENCES}

1. Petty RE, Southwood TR, Baum J, Bhettay E, Glass DN, Manners $\mathrm{P}$, et al. Revision of the proposed classification criteria for juvenile idiopathic arthritis: Durban, 1997. J Rheumatol 1998;25:1991-4.

2. Petty RE, Southwood TR, Manners P, Baum J, Glass DN, Goldenberg J, et al; International League of Associations for Rheumatology. International League of Associations for Rheumatology classification of juvenile idiopathic arthritis: second revision, Edmonton, 2001. J Rheumatol 2004;31:390-2.

3. Martini A. Are the number of joints involved or the presence of psoriasis still useful tools to identify homogeneous disease entities in juvenile idiopathic arthritis? J Rheumatol 2003;30:1900-3.

4. Martini A. It is time to rethink juvenile idiopathic arthritis classification and nomenclature. Ann Rheum Dis 2012;71:1437-9.

5. Ravelli A, Felici E, Magni-Manzoni S, Pistorio A, Novarini C, Bozzola E, et al. Patients with antinuclear antibody-positive juvenile idiopathic arthritis constitute a homogeneous subgroup irrespective of the course of joint disease. Arthritis Rheum 2005;52:826-32.

6. Ravelli A, Varnier GC, Oliveira S, Castell E, Arguedas O, Magnani A, et al. Antinuclear antibody-positive patients should be grouped as a separate category in the classification of juvenile idiopathic arthritis. Arthritis Rheum 2011;63:267-75.

7. Hinks A, Bowes J, Cobb J, Ainsworth HC, Marion MC, Comeau ME, et al. Fine-mapping the MHC locus in juvenile idiopathic arthritis (JIA) reveals genetic heterogeneity corresponding to 
distinct adult inflammatory arthritic diseases. Ann Rheum Dis 2017;76:765-72.

8. Nigrovic PA, Raychaudhuri S, Thompson SD. Review: genetics and the classification of arthritis in adults and children. Arthritis Rheumatol 2018;70:7-17.

9. Eng SW, Duong TT, Rosenberg AM, Morris Q, Yeung RS; REACCH OUT and BBOP Research Consortia. The biologic basis of clinical heterogeneity in juvenile idiopathic arthritis. Arthritis Rheumatol 2014;66:3463-75.

10. Ombrello MJ, Remmers EF, Tachmazidou I, Grom A, Foell D, Haas JP, et al; International Childhood Arthritis Genetics (INCHARGE) Consortium. HLA-DRB $1 * 11$ and variants of the MHC class II locus are strong risk factors for systemic juvenile idiopathic arthritis. Proc Natl Acad Sci U S A 2015;112:15970-5.

11. Ombrello MJ, Arthur VL, Remmers EF, Hinks A, Tachmazidou I, Grom AA, et al. Genetic architecture distinguishes systemic juvenile idiopathic arthritis from other forms of juvenile idiopathic arthritis: clinical and therapeutic implications. Ann Rheum Dis 2017; 76:906-13.

12. Delbecq AL, Van de Ven AH, Gustafson DH. Group techniques for program planning. A guide to nominal group and Delphi processes. Glenview, IL: Scott, Foresman; 1975.

13. Ruperto N, Meiorin S, Iusan SM, Ravelli A, Pistorio A, for the Paediatric Rheumatology International Trials Organisation (PRINTO). Consensus procedures and their role in pediatric rheumatology. Curr Rheumatol Rep 2008;10:142-6.

14. Ruperto N, Ravelli A, Murray KJ, Lovell DJ, Andersson-Gare B, Feldman BM, et al; Paediatric Rheumatology International Trials Organization (PRINTO); Pediatric Rheumatology Collaborative Study Group (PRCSG). Preliminary core sets of measures for disease activity and damage assessment in juvenile systemic lupus erythematosus and juvenile dermatomyositis. Rheumatology 2003;42:1452-9.

15. Ruperto N, Ravelli A, Cuttica R, Espada G, Ozen S, Porras O, et al; Pediatric Rheumatology International Trials Organization (PRINTO); Pediatric Rheumatology Collaborative Study Group (PRCSG). The Pediatric Rheumatology International Trials Organization criteria for the evaluation of response to therapy in juvenile systemic lupus erythematosus: prospective validation of the disease activity core set. Arthritis Rheum 2005;52:2854-64.

16. Ruperto N, Ravelli A, Oliveira S, Alessio M, Mihaylova D, Pasic S, et al; Pediatric Rheumatology International Trials Organization. The Pediatric Rheumatology International Trials Organization/American College of Rheumatology provisional criteria for the evaluation of response to therapy in juvenile systemic lupus erythematosus: prospective validation of the definition of improvement. Arthritis Rheum 2006;55:355-63.

17. Felson DT, Anderson JJ, Boers M, Bombardier C, Furst D, Goldsmith C, et al. American College of Rheumatology preliminary definition of improvement in rheumatoid arthritis. Arthritis Rheum 1995;38:727-35.

18. Giannini EH, Ruperto N, Ravelli A, Lovell DJ, Felson DT, Martini A. Preliminary definition of improvement in juvenile arthritis. Arthritis Rheum 1997;40:1202-9.

19. Ruperto N, Ravelli A, Falcini F, Lepore L, De Sanctis R, Zulian F, et al. Performance of the preliminary definition of improvement in juvenile chronic arthritis patients treated with methotrexate. Ann Rheum Dis 1998;57:38-41.

20. Rider LG, Giannini EH, Brunner HI, Ruperto N, James-Newton L, Reed AM, et al; International Myositis Assessment and Clinical Studies Group. International consensus on preliminary definitions of improvement in adult and juvenile myositis. Arthritis Rheum 2004;50:2281-90.

21. Ruperto N, Ravelli A, Pistorio A, Ferriani V, Calvo I, Ganser G, et al. The provisional Pediatric Rheumatology International Trial
Organization/American College of Rheumatology/European League Against Rheumatism disease activity core set for the evaluation of response to therapy in juvenile dermatomyositis: a prospective validation study. Arthritis Rheum 2008;59:4-13.

22. Jennette JC, Falk RJ, Andrassy K, Bacon PA, Churg J, Gross WL, et al. Nomenclature of systemic vasculitides: proposal of an international consensus conference. Arthritis Rheum 1994; 37:187-92.

23. Yamaguchi M, Ohta A, Tsunematsu T, Kasukawa R, Mizushima Y, Kashiwagi H, et al. Preliminary criteria for classification of adult Still's disease. J Rheumatol 1992;19:424-30.

24. Van der Linden S, Valkenburg HA, Cats A. Evaluation of diagnostic criteria for ankylosing spondylitis. A proposal for modification of the New York criteria. Arthritis Rheum 1984;27:361-8.

25. Lambert RG, Bakker PA, Van der Heijde D, Weber U, Rudwaleit M, Hermann KG, et al. Defining active sacroiliitis on MRI for classification of axial spondyloarthritis: update by the ASAS MRI working group. Ann Rheum Dis 2016;75:1958-63.

26. Sieper J, Van der Heijde D, Landewe R, Brandt J, Burgos-Vagas R, Collantes-Estevez E, et al. New criteria for inflammatory back pain in patients with chronic back pain: a real patient exercise by experts from the Assessment of SpondyloArthritis international Society (ASAS). Ann Rheum Dis 2009;68:784-8.

27. Barnes MG, Grom AA, Thompson SD, Griffin TA, Luyrink LK, Colbert RA, et al. Biologic similarities based on age at onset in oligoarticular and polyarticular subtypes of juvenile idiopathic arthritis. Arthritis Rheum 2010;62:3249-58.

28. Castaneda S, Blanco R, Gonzalez-Gay MA. Adult-onset Still's disease: advances in the treatment. Best Pract Res Clin Rheumatol 2016;30:222-38.

29. Martini A. Systemic juvenile idiopathic arthritis. Autoimmun Rev 2012;12:56-9.

30. Masters SL, Simon A, Aksentijevich I, Kastner DL. Horror autoinflammaticus: the molecular pathophysiology of autoinflammatory disease (*). Annu Rev Immunol 2009;27:621-68

31. Gattorno M, Piccini A, Lasiglie D, Tassi S, Brisca G, Carta S, et al. The pattern of response to anti-interleukin-1 treatment distinguishes two subsets of patients with systemic-onset juvenile idiopathic arthritis. Arthritis Rheum 2008;58:1505-15.

32. Arthur VL, Shuldiner E, Remmers EF, Hinks A, Grom AA, Foell D, et al. IL1RN variation influences both disease susceptibility and response to recombinant human interleukin-1 receptor antagonist therapy in systemic juvenile idiopathic arthritis. Arthritis Rheumatol 2018;70:1319-30.

33. Guzman J, Oen K, Tucker LB, Huber AM, Shiff N, Boire G, et al; ReACCh-Out investigators. The outcomes of juvenile idiopathic arthritis in children managed with contemporary treatments: results from the ReACCh-Out cohort. Ann Rheum Dis 2015;74:1854-60.

34. Van Rossum MA, Van Soesbergen RM, de Kort S, ten Cate R, Zwinderman AH, de Jong B, et al. Anti-cyclic citrullinated peptide (anti-CCP) antibodies in children with juvenile idiopathic arthritis. J Rheumatol 2003;30:825-8.

35. Gilliam BE, Chauhan AK, Moore TL. Evaluation of anti-citrullinated type II collagen and anti-citrullinated vimentin antibodies in patients with juvenile idiopathic arthritis. Pediatr Rheumatol Online J 2013;11:31.

36. Aggarwal A. Role of autoantibody testing. Best Pract Res Clin Rheumatol 2014;28:907-20.

37. Colbert RA. Classification of juvenile spondyloarthritis: enthesitis-related arthritis and beyond. Nat Rev Rheumatol 2010;6:477-85.

38. Tse SM, Laxer RM. New advances in juvenile spondyloarthritis. Nat Rev Rheumatol 2012;8:269-79.

39. Burgos-Vargas R, Pacheco-Tena C, Vazquez-Mellado J. Juvenile-onset spondyloarthropathies. Rheum Dis Clin North Am 1997;23:569-98. 